\title{
Pulmonary Fistula, CTCAE
}

National Cancer Institute

\section{Source}

National Cancer Institute. Pulmonary Fistula, CT CAE. NCI Thesaurus. Code C143791.

A disorder characterized by an abnormal communication between the lung and another organ or anatomic site. 